\title{
MAPEAMENTO DE HABITATS MARINHOS DA PLATAFORMA CONTINENTAL INTERNA DA PRAIA DE IRACEMA - FORTALEZA - CEARÁ
}

\author{
MAPPING OF MARINE HABITATS OF THE INTERNAL CONTINENTAL SHELFOF THE \\ BEACH OFIRACEMA - FORTALEZA - CEARÁ
}

\begin{abstract}
Lidriana de Souza PINHEIRO ${ }^{1}$, Francisco Gleidson GASTÃO ${ }^{1}$, Yan Thé Cardozo LOUSADA $^{1}$, Wilson FRANKLIN JÚNIOR ${ }^{2}$, Mônica Pimenta Novaes CASTELO BRANCO ${ }^{1}$

${ }^{1}$ Laboratório de Oceanografia Geológica (LOG)/Instituto de Ciências do Mar (Labomar)/Universidade Federal do Ceará - UFC. Av. da Abolição, 3207 - Meireles - Fortaleza - CE. Emails: lidriana@ufc.br; f.gleidson@ufc.br; yanthe96@hotmail.com; castelobranco.monica@gmail.com

${ }^{2}$ Laboratório de Zoobentos/Instituto de Ciências do Mar (Labomar)/Universidade Federal do Ceará - UFC. Av. da Abolição, 3207 -
\end{abstract} Meireles - Fortaleza - CE. Email: wfranklinjr@gmail.com

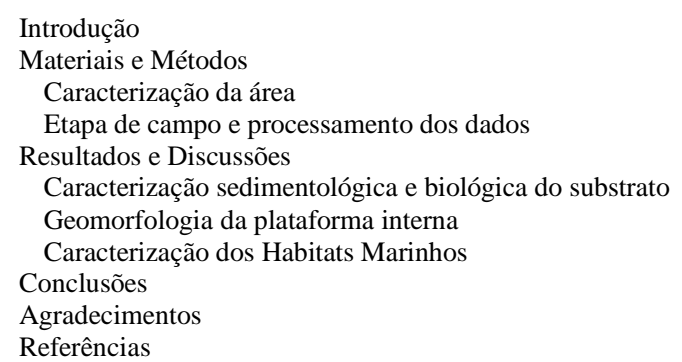

RESUMO - O presente trabalho tem como objetivo caracterizar os habitats marinhos da plataforma continental interna da Praia de Iracema - Fortaleza/CE, a partir da análise integrada do substrato bentônico. Para isto, foi feita a batimetria de fundo, sedimentologia e a fauna e flora bentônica dos fragmentos de paisagens, com base em dados coletados em campo e analisados em laboratório. As análises granulométricas realizadas nas 20 amostras apontaram para uma distribuição das frações: cascalho, areia média, areia fina e muito fina e frações biogênicas. A classe das areias está em pontos isolados, com um percentual de 90 a $100 \%$, contendo algumas percentagens de mica. As areias médias ocorrem na porção NW da área. Os depósitos biogênicos correspondem a sedimentos constituídos por fragmentos de animais marinhos e algas calcárias. O destaque da biodiversidade foi encontrado na Estação 3, onde pode-se verificar exemplares dos Filos Echinodermata, Mollusca, Porifera e Annelida. Nas amostras essencialmente arenosas, foi observada a presença de organismos pertencentes ao Filo Mollusca - Classe Bivalvia. A interação dos dados bióticos e abióticos (sedimentológicos e batimétricos) permitiu mapear quatro habitats marinhos, enquadrados nos Níveis 1 e 2, códigos A (Marine Habitats) e A1 (Littoral Rock andother hard substrata), da classificação proposta pela EUNIS, 2012.

Palavras-chave: Habitats Marinhos, Plataforma Continental, Sedimentologia, Batimetria.

ABSTRACT - The present work has the objective of characterizing marine habitats of the continental shelf of Iracema beach in Fortaleza, Ceara, from the integrated analysis of the benthic substract. This characterization was based on the background bathymetry, sedimentology and benthic fauna and flora of the landscape fragments of data collected on the site and analyzed in the laboratory. The particle size analyzes on the 20 field samples indicated a fraction distribution of gravel, medium, fine and very fine sand and biogenic fractions. The sands class are in isolated spots, with a percentage of 90 to $100 \%$, with some containing mica The medium sand occurs in the NW portion of the area. The biogenic deposits correspond to sediments consisting of fragments of marine animals and calcareous algae. The biodiversity highlight of the biodiversity was found in Station3, where were found examples of the Echinodermata, Mollusca, Porifera, and Annelida phyla. In the samples substantially sandy, it was observed the presence of organisms belonging to the phylum Mollusca - Class Bivalvia. The biotic and abiotic data interaction (sedimentological and bathymetric) allowed the mapping of four marine habitats, classified in Levels1and 2, codes A (Marine Habitats) and A1 (Littoral Rock and other hard substrata), of the classification proposed by EUNIS, 2012.

Keywords: Marine Habitats, Continental Shelf, Sedimentology, Bathymetry.

\section{INTRODUÇÃO}

Habitat é um termo que define o espaço físico de vivência de organismos (animais e vegetais), distribuídos por um conjunto de fatores distintos do ambiente (Begon et al., 1990). A European Nature Information System (EUNIS, 2012), que corresponde ao sistema de informações de referência para o desenvolvimento de trabalhos de ecologia e conservação da natureza, classifica hierarquiacamente habitats a fatores abióticos e bióticos. Segundo a EUNIS (2012), habitats correspondem a comunidades de animais e plantas que, em conjunto com determinados fatores abióticos, formam uma unidade homogênea com uma determinada dimensão, e permite caracterizar o ambiente biótico. Ainda, segundo esse Sistema, um habitat é selecionado a partir de um conjunto de parâmetros físicos e biológicos (e.g. tipos de substrato, intensidade da luz no fundo, zonas 
biológicas, usos e impactos da atividade humana, e comunidades dominantes).

O Reino Unido e a Irlanda foram os pioneiros na classificação dos habitats marinhos, apresentando um sistema de classificação capaz de ajudar a gestão e a conservação, principalmente, em relação aos habitats marinhos bentônicos (habitats do litoral e dos fundos marinhos e suas comunidades de espécies associadas) (Connor et. al., 2004).

Dias et al (2004) definem os habitats bentônicos a partir do conjunto de fatores geológicos associados às características físicoquímicas das massas de água. Os fatores correlacionados com a geologia desempenham um papel fundamental na estabilidade e composição do leito marinho, possuindo uma variação muito menor quando comparados com parâmetros físico-químicos atuantes nos habitats marinhos.

Isso faz com que as informações geológicas do substrato marinho sejam mais relevantes na predição de habitats (Möller et al., 1985; Coleman et al., 1997; Dias et al., 2004; Diaz et al, 2004; Secchin, 2011).

Dessa maneira, a fisiografia, a profundidade, a dureza do substrato, a textura sedimentar, a geomorfologia e a biologia são amplamente utilizadas para classificar os habitats no fundo marinhos (Greene et al., 2007). Entretanto, as pesquisas desenvolvidas na plataforma continental de Fortaleza, muitas vezes, limitam-se na caracterização faciológica do substrato marinho isoladamente ou na descrição de determinadas espécies animais e/ou vegetais, sem a realização de um processo interativo entre os componentes abióticos e os bióticos (Kempf et al., 1970; Morais, 1972, 1981 e 2010; Freire et al., 1984 e 1993; Freire, 1985, Freire \& Cavalcanti, 1998; Coutinho, 1996; Maia et al., 1997, 2008, Soares \& Morais, 2009; Rebouças, 2010; Monteiro, 2011; Soares, 2012; Lima et al., 2018). Estudos que integram o meio biótico e abiótico são imprescindíveis para fins de conservação e manejo de áreas marinhas, principalmente aquelas que sofrem pressões dos impactos antropogênicos, a exemplo de áreas marinhas adjacentes a grandes centros urbanos, como é o caso de Fortaleza.

Em face do exposto, o presente estudo tem como objetivo principal realizar um mapeamento do ambiente marinho através da descrição dos habitats, destacando as características dos parâmetros geológicos do substrato marinho da plataforma interna de Fortaleza (Praia de Iracema), assim como a descrição dos componentes bióticos presentes no substrato marinho em questão. Realizando, portanto, uma interpretação interativa entre os componentes abióticos e bióticos.

\section{Caracterização da Área}

A Praia de Iracema está localizada na porção mais a oeste da orla central da cidade de Fortaleza, correspondendo, portanto, à área de influência do Porto do Mucuripe, construído na década de 50. Fortaleza é a capital do Estado do Ceará, com uma população de 2,643 milhões de habitantes (Ipece, 2018) e uma orla com 34,2 km de extensão. A área de estudo encontra-se limitada pela histórica Ponte dos Ingleses, Praia de Iracema, e o espigão situado defronte a Avenida Rui Barbosa, Praia do Meireles (Figura 1). Apresenta urbanização consolidada, e em alguns trechos recebe descargas de efluentes de esgotos domésticos que são transportados por riachos e/ou por ligações clandestinas nas galerias pluviais distribuídas ao longo da cidade, de acordo com o Projeto Orla (Unidade II, Trecho 1 a 4) (Brasil, 2006). Os principais usos são para as atividades balneárias, esportivas na areia e no mar, incluindo a pesca.

\section{Etapa de campo e processamentos dos dados}

Foi delimitada uma área de $1,7 \mathrm{~km} 2$, com escala de 1: 10.0000 contemplando os substratos consolidados (beachrocks, plataformas de abrasão e obras de proteção) e inconsolidados.

No dia 30 de novembro de 2017, foi realizado levantamento batimétrico por meio de ecosonda (modelo GARMIN) acoplado a um receptor GPS, registrando informações (dados brutos), em protocolo NMEA (National Marine Electronics Association) ou sentenças relacionadas às coordenadas geográficas dos pontos, a profundidade da lâmina d'água e à hora dos registros, todas em formato do Código Padrão Americano conhecido por ASCII para o laptop. Os dados foram corrigidos ao nível reduzido (zero hidrográfico) baseado na tábua de maré do Porto do Mucuripe, disponibilizada no site da Diretoria de Hidrografia e Navegação (DHN) da Marinha do Brasil. A correção de maré é feita a partir de uma equação gerada pela curva correspondente ao intervalo do levantamento (Figura 2). 


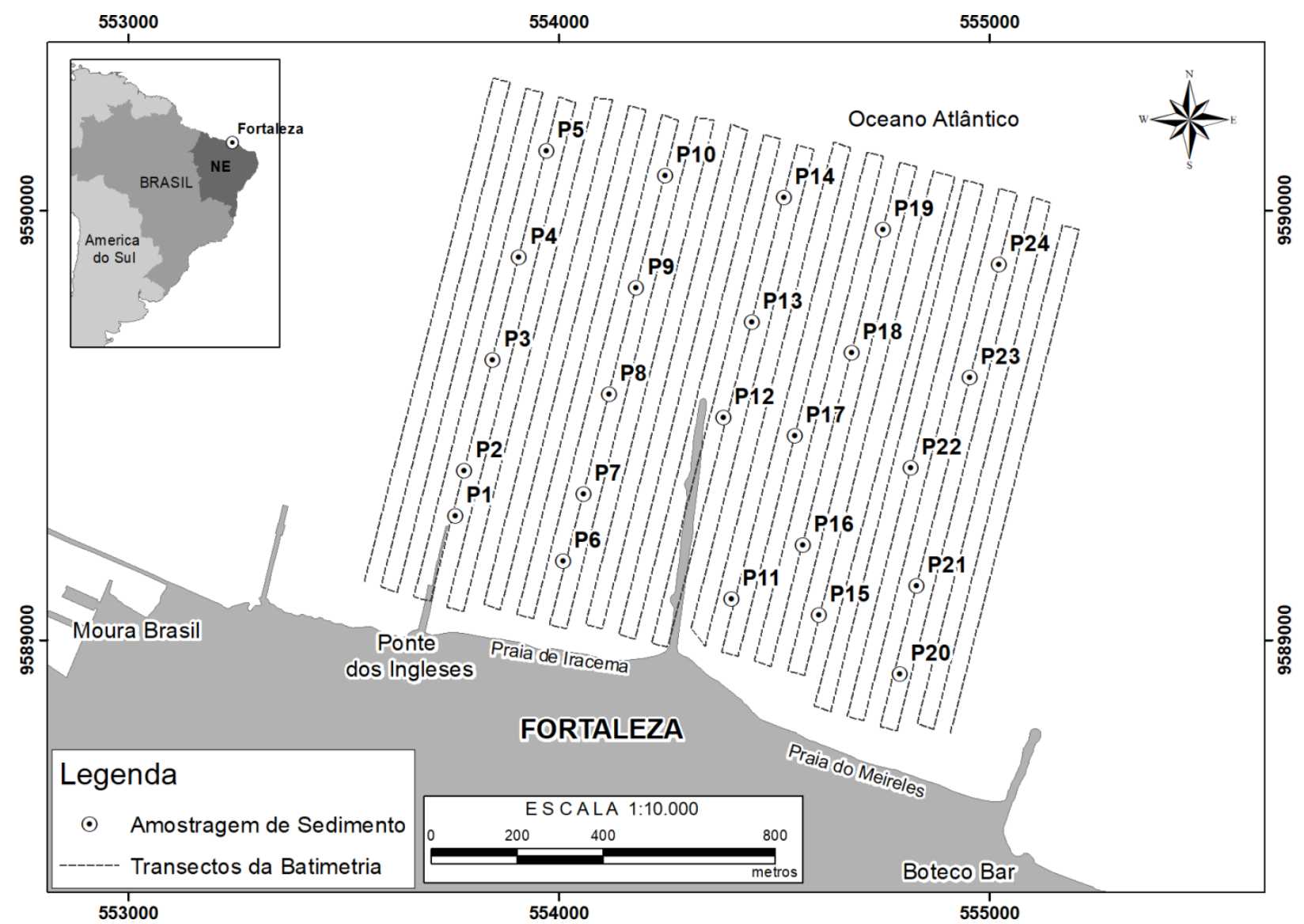

Figura 1. Mapa de localização da área e malhas amostrais para coleta do substrato marinho e dos transectos da batimétrica.

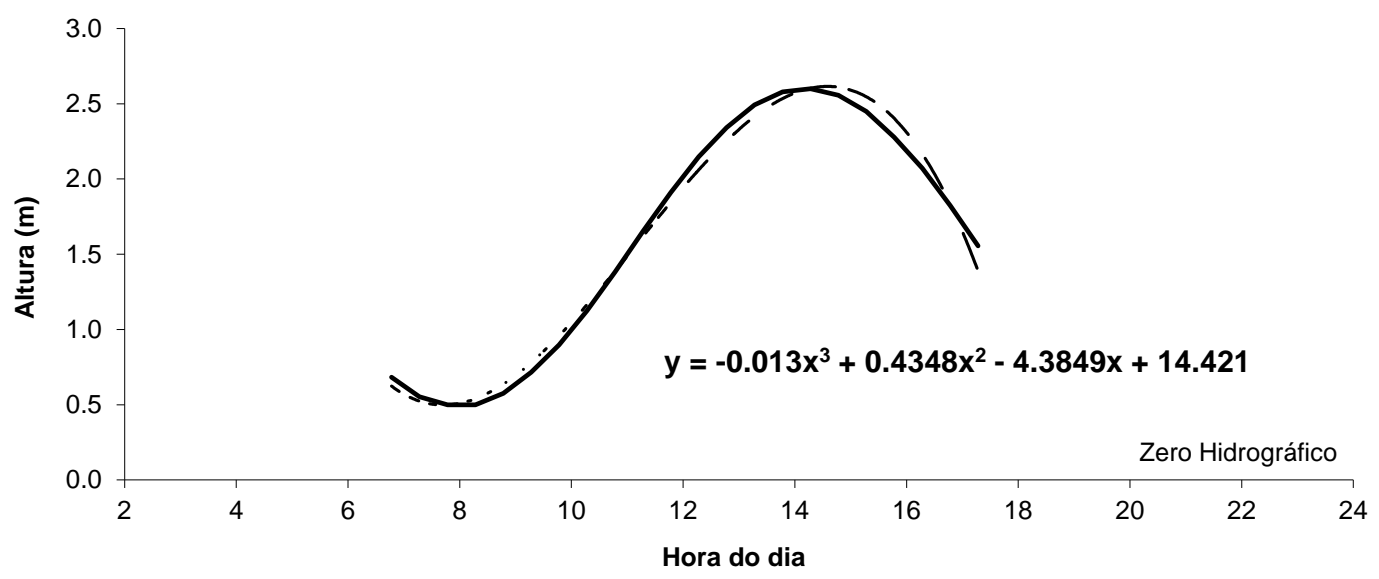

Figura 2. Curva da maré (linha contínua) e equação gerada pela linha de tendência (linha pontilhada) para o intervalo de levantamento do dia 30/11/17, das 7:00 às 17:30hs.

Foi feita ainda a exclusão dos pontos com cotas e valores anômalos (outliers) ao levantamento, que pudessem causar uma distorção na krigagem dos dados na etapa seguinte. Estes pontos anômalos são gerados por eventuais perturbações das ações hidrodinâmicas das ondas e marés na hora do levantamento.

O geoprocessamento dos dados XYZ ocorreu por intermédio do software Quantum GIS, onde foi realizada a interpolação dos dados através do método estatístico krigagem, $\mathrm{O}$ mapa batimétrico foi elaborado utilizando o datum horizontal WGS84 Zona 24 Sul (sistema de coordenadas UTM).

A partir dos dados batimétricos, gerado pelo modelo raster, foi possível elaborar quatro perfis topográficos em forma de gráficos no software Quantum GIS, posteriormente exportados para o formato Excel. Através dos perfis topo batimétricos foi possível aferir as condições de fundo, identificar determinadas formações e verificar 
a declividade da plataforma continental.

As amostras do substrato marinho foram coletadas por meio de draga Van Veen. O planejamento indicou 24 pontos a serem amostrados, entretanto, em função da natureza do substrato, constituídos por rochas consolidadas, não foi possível a coleta de todos os pontos planejados. Sendo, portanto, coletada apenas em 20 pontos (\# 3, 4, 5, 6, 7, 8, 10, 11, 12, 13, 14, 15, 16, 17, 18, 19, 20, 22, 23 e 24) (Figura 1). Em campo, essas amostras foram descritas quanto as tenças, com base na proposta de Dias, 1996. No Laboratório de Oceanografia Geológica (LOG) do Instituto de Ciências do Mar (LABOMAR/UFC) foram realizadas as análises: granulométrica, determinação de carbonato de cálcio e de matéria orgânica, pelos métodos propostos por Suguio (1973),
Calcímetro de Bernard modificado (Soares, 2017) e Davies (1997), respectivamente.

A descrição dos componentes bióticos (autóctones) aconteceu em dois momentos: o primeiro imediatamente após a coleta e o segundo após o processo de peneiramento mecânico das amostras. Os componentes bióticos foram descritos e classificados no nível dos grandes grupos (Filos), por intermédio de observações a olho nu, por pesquisadores do Laboratório de Zoobentos do Instituto de Ciências do Mar (LABOMAR/UFC).

A caracterização dos habitats marinhos aconteceu pela interpolação das características abióticas (sedimentologia e batimetria) do ambiente e os dados classificatórios dos componentes bióticos. Sendo classificados segundo o Sistema de Informações EUNIS (2012).

\section{RESULTADOS E DISCUSSÃO}

\section{Caracterização Sedimentológica e Biológica do Substrato}

A descrição das tenças reuniu as amostras em seis grupos: Coral calcário (\#3); Areia média (\#4, 5, 6, 7, 10, 15, 17, 18); Areia fina (\#8, 13, 14, 19, 20, 23, 24); Areias finas com algas calcárias (\# 11, 12, 16); Rocha/Algas calcárias (\#22) e um último grupo definido por Rocha (dura ou calcárias), atribuído às amostras \#1, 2, 9 e 21, que em função da impossibilidade de coleta foram definidas como um substrato consolidado (rochoso). Portanto, fica visível o predomínio de um substrato arenoso (areia média e fina).

A sedimentação no domínio da plataforma continental interna da área estudada caracteriza-se por depósitos biogênicos, muito provavelmente alóctones, e terrígenos. Os depósitos biogênicos representam os sedimentos compostos por carapaças de organismos ou biodetritos constituídos, em sua maioria, por algas calcárias e fragmentos de moluscos (bivalves).

As frações predominantes na área foram: Areia média (\#4 e 5), areia fina (\#6, 11, 12, 15 e 16) e areia muito fina $(\# 7,8,10,13,14,17,18,19,20$, 23 e 24). Pode-se observar o predomínio de areias finas e muito finas espacialmente distribuídas na área. Enquanto as areias de diâmetro 0,250 a 0,500 $\mathrm{mm}$, areias médias, ocorrem restritamente na porção NW da área, provavelmente, correspondendo a bancos arenosos localizados na isóbata de $-10 \mathrm{~m}$, defronte a linha de costa de Fortaleza (Soares, 2012).
Nas estações \#11, \#12 e \#16 a areia fina foi predominante. Entretanto, as amostras coletadas retratam uma associação do substrato misto, constituído por areia siliciclástica fina com organismos e/ou fragmentos de carapaças de organismos marinhos (Filo Mollusca da classe Bivalvia) e algas calcárias, imprimindo, dessa maneira, um aspecto cascalhoso ( $>2 \mathrm{~mm})$.

A classe das areias ( $>64 \mu \mathrm{m}$ a $<2 \mathrm{~mm}$ ) estão em pontos isolados (\#4, 5, 6, 7, 8, 10,13, 14, 15, 17, $18,19,20,23$ e 24) com um percentual de 80 a $100 \%$ da fração areia, contendo em algumas frações plaquetas de mica e grãos de mineralogia acessória (minerais pesados), mostrando forte influência da sedimentação terrígena na área. Os depósitos terrígenos correspondem as acumulações tipicamente caracterizadas por grãos de quartzo (sílica - SiO2), micas (silicatos (X2Y46Z8O20(OH,F)4), feldspato - (silicatos (K, Na $\mathrm{Ca})(\mathrm{Si}, \mathrm{Al}) 4 \mathrm{O} 8)$ e raros minerais acessórios (minerais pesados).

Em relação ao grau de seleção, as amostras foram distribuídas em três grupos: a) sedimentos muito pobremente selecionados (\#8, $10,12,13,16,19,20$ e 23), b) pobremente selecionados (\#7, 11, 14, 17, 18 e 24), e c) os sedimentos moderadamente selecionados (\#4, 5, 6 e 15). Pôde-se observar o predomínio de sedimentos muito pobremente selecionados a pobremente selecionados, indicando a presença de sedimentos caracterizados por natureza bimodal. Esta característica ocorre quando duas modas distintas, de acordo com o tamanho do 
grão, são encontradas na mesma amostra (Suguio, 1973). As amostras de natureza bimodal da área estudada são compostas por um conjunto de sedimentos arenosos, representados pelos sedi-mentos terrígenos, juntamente com sedimentos cascalhosos (bioclástico) ou lamosos. Situação essa bem caracterizada nas estações \#11, 12 e 16, descritas anteriormente.

Os teores de carbonato de cálcio (CaCO3) oscilaram de 1,47\% (\#4) a 38,50\% de CaCO3 (\#3). De maneira geral, foram identificados quatro grupos, envolvendo as seguintes variações de teores de CaCO3: teores $<10 \%$ (\#4 e 5); teores de 10 a $20 \%(\# 6,13,14,15,16,17$, 18, 19, 23 e 24), teores entre 20 a 30\% (\#7, 8, $10,11,12$ e 20$)$ e teores $>30 \%$ (\#3 e 22). Essas variações estão associadas à presença/ausência de fragmentos de conchas e algas calcárias nas amostras, tanto de diâmetros macroscópicos quanto microscópicos. Os constituintes microscópicos foram caracterizados por fragmentos, não maiores do que $0,2 \mathrm{~mm}$, de conchas (Filo Mollusca da classe Bivalvia, (Figuras 3A e 3D), algas calcárias, carapaças de foraminíferos (Figuras 3C), espículas de esponjas (Figura 3B) e fragmentos de briozoários, ouriço (Figura 3E) e crustáceo (Figura 3F).

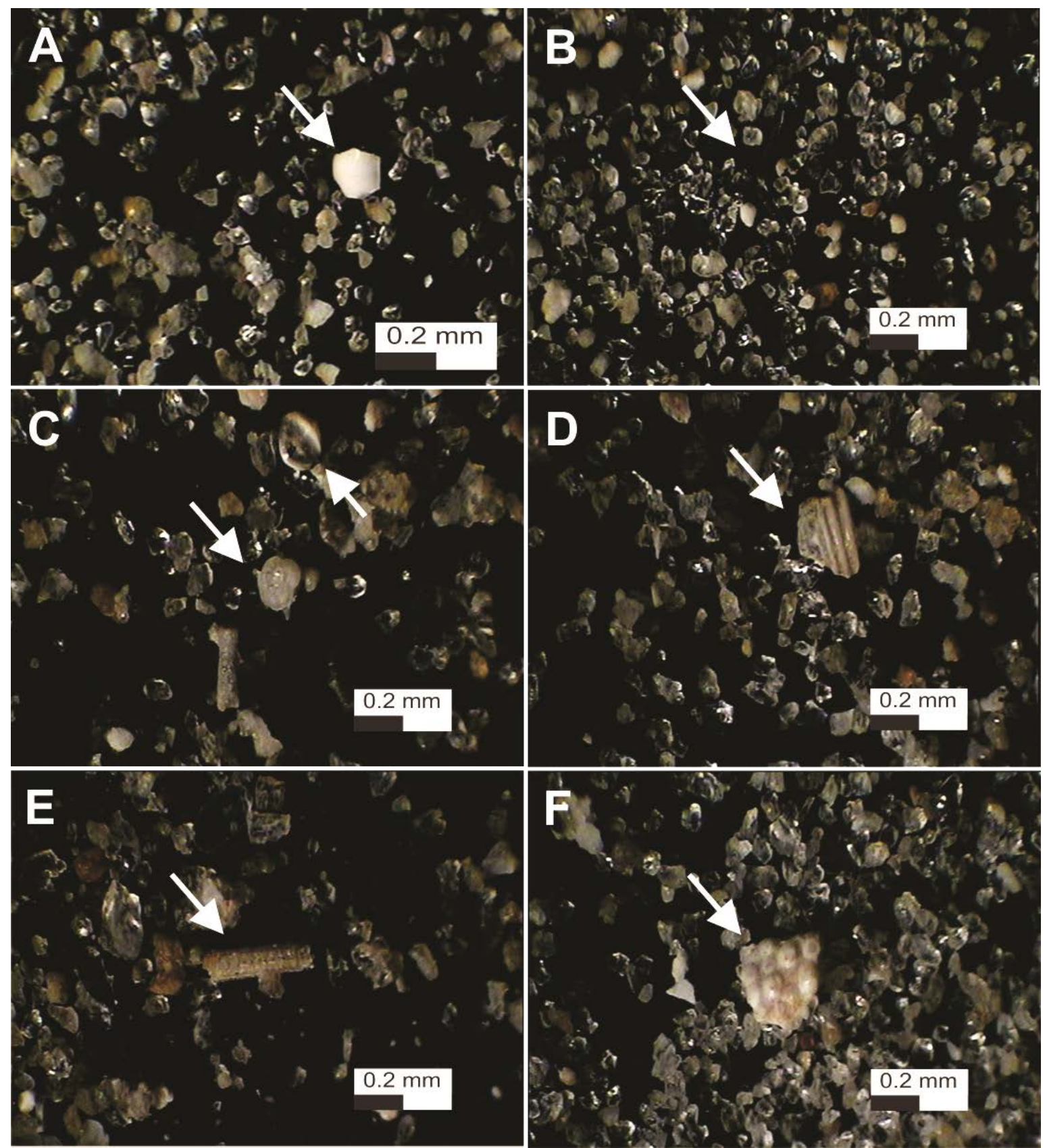

Figura 3- Biodetritos entre grãos de quartzo nas amostras que deram teores acima de $20 \%$ de CaCO3. A - Fragmento de concha de bivalve da estação \#7. B - Espícula de esponja da estação \#7. C - Foraminíferos da estação \#8. D Fragmento de bivalve da estação \#8. E - Fragmento de espinho de ouriço da estação \#10. F - Fragmento de crustáceo da estação \#8. 
De acordo com Saliot (1994 apud Catanzaro 2002) os percentuais de matéria orgânica variam de 1 a $11 \%$ nos lagos, 0,7 a 5\% nos estuários, e 0,28 a 3,2\% na plataforma continental.

A partir desses índices é possível notar que os sedimentos amostrados apresentam altos percentuais de matéria orgânica, variando de 0,3 a $9,981 \%$.

O valor mais elevado (9,981\%) ocorre na amostra \#12, provavelmente, associado a um ambiente de baixa energia hidrodinâmica, área abrigada pela implantação do espigão, indicativo de ambiente redutor ou de baixa degradação de matéria orgânica.

Portanto, caracterizado por ambiente de águas calmas e de baixa velocidade de correntes, e consequentemente, maior concentração de matéria orgânica proveniente da concentração de organismos (animais e vegetais) e/ou de lançamento de efluentes (pluviais ou domésticos clandestinos).

Nas estações \#3, 11 e 22 as concentrações variam de 3,0 a 6\%. Embora estas estações não

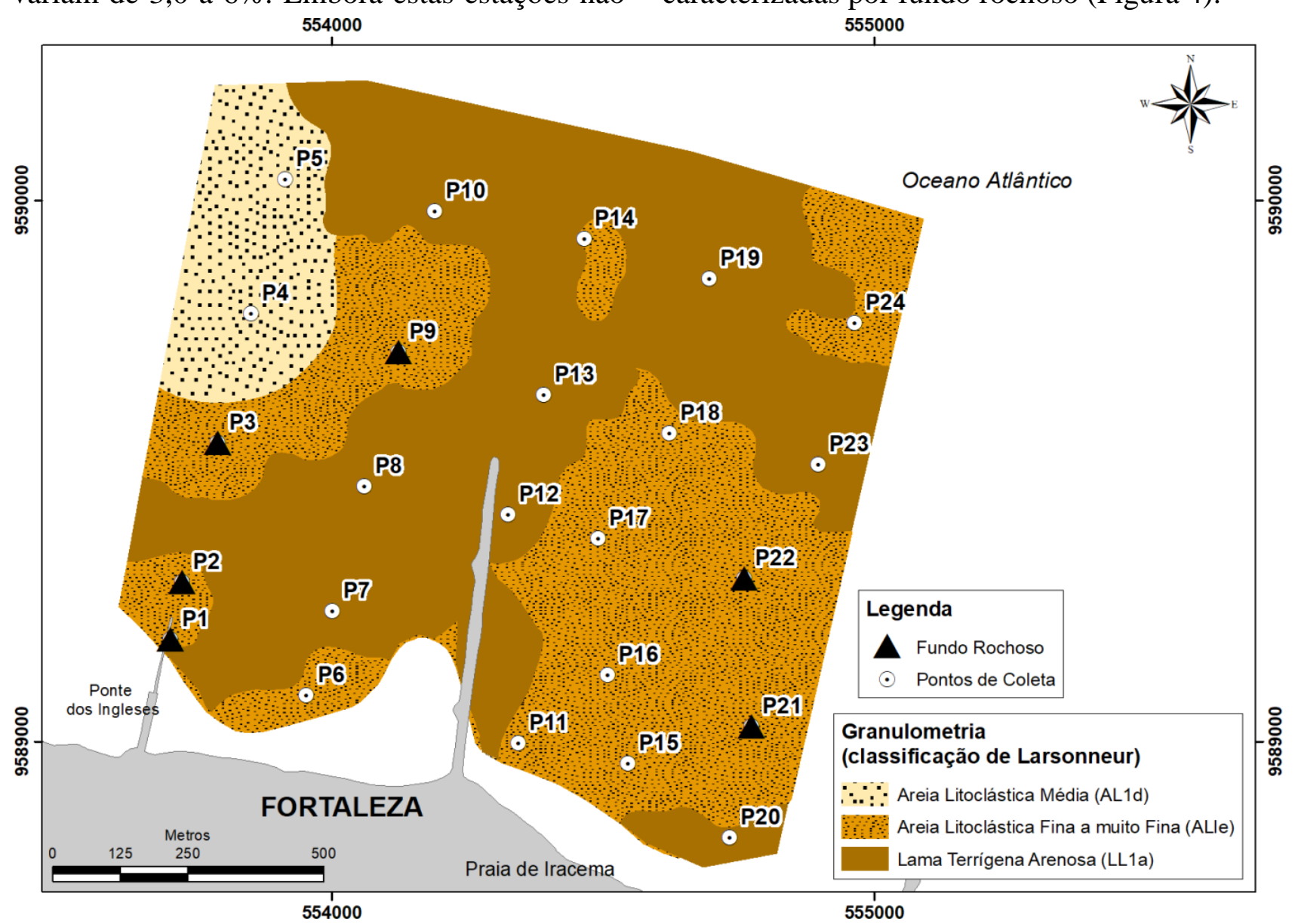

Figura 4- Mapa de classificação granulométrica dos sedimentos baseado na classificação de Larsounner (1977), modificada por Dias (1996).

As estações \#3, \#11, \#12, \#16 e \#22, foram caracterizadas por um enriquecimento de estejam em área mais abrigada que a estação \#12, elas são compostas por biodetritos, desde algas calcárias a conchas do Filo Molusca.

Segundo a classificação de Larsonneur (1977) modificada por Dias (1996) as amostras foram identificadas como: Areias Litoclásticas Médias (AL1d) caracterizadas por grãos variando de 0,25 a $5 \mathrm{~mm}$ e teores de carbonato de cálcio $<30 \%$, mapeadas nas estações \#4 e \#5; Areias Litoclásticas Finas a Muito Finas (ALIe), cujos grãos assumem diâmetros de 0,05 a $0,25 \mathrm{~mm}$ e teores de carbonato de cálcio < $30 \%$, presentes nas estações \# $6,11,14,15,16$, 17, 18 e 24 e Lamas Terrígenas Arenosas (LL1a), com percentuais de Lama variando de 25 a 75\% e teores de carbonato de cálcio (CaCO3) < 30\%, encontradas nas estações \# 7 , $8,10,12,13,19,20$ e 23.

Vale ressaltar que para essa distribuição das classes granulométricas de Larsonneur na área estudada, estão excluídas as amostras das estações \#1, 2, 3, 9, 21 e 22, todas caracterizadas por fundo rochoso (Figura 4).

555000

depósitos biogênicos, com presença de coral calcário e uma associação de componentes 
terrígenos com fragmentos de algas calcárias e rochas calcárias.

A Tabela 1 e a Figura 5 mostram a descrição dos componentes bióticos e ilustração dessas amostras.

O destaque foi para a amostra \#3, onde a biodiversidade foi marcada por representantes dos Filos Echinodermata, Mollusca, Porifera e Annelida. Foi observada a presença de organismos bentônicos, principalmente na classe das areias (diâmetro de 0,500 a 1,00mm). Em geral, esses componentes foram classificados como bentônicos pertencentes ao Filo Mollusca - Classe Bivalvia.

Tabela 1. Descrição dos componentes bióticos.

\begin{tabular}{l|l}
\hline ESTAÇÕES & \multicolumn{1}{c}{ DESCRIÇÃO BIOLÓGICA } \\
\hline$\# \mathbf{3}$ & $\begin{array}{l}\text { - Presença de “Serpentes do Mar” (Filo Echinodermata - Classe Ophiuroidea). } \\
\text { - Exemplares macroscópicos do Filo Mollusca (Classe Bivalvia), Esponjas (Filo Porifera), } \\
\text { algas calcárias (rodolitos) e Filo Annelida (Classe Polychaeta). }\end{array}$ \\
\hline \# 11 e 12 & - Diversos fragmentos de algas (Divisão Rodophyta e Phaeophyta). \\
\hline \# 16 & $\begin{array}{l}\text { - Presença de rodolitos (algas vermelhas - calcárias), esponjas (Filo Porifera) e macro algas } \\
\text { como ramos de algas vermelhas (Rodophyta) e pardas (Phaeophyta). }\end{array}$ \\
\hline \#22 & $\begin{array}{l}\text { Presença de carapaças de conchas do Filo Molusca (Classe Bivalvia) de tamanho de 0,5 a } \\
1,5 \mathrm{~cm} .\end{array}$ \\
\hline
\end{tabular}

\section{Geomorfologia da Plataforma Interna}

A morfologia da área é considerada heterogênea em função da presença de recifes submersos, que na maioria das vezes, estão distribuídos nas áreas planas que constituem os substratos arenosos.

Com exceção na porção NW da área, próximo as estações \#4 e \#5, onde migram lateralmente para relevo ondulado de natureza arenosa, classificado por Larsounner (1977), modificado por Dias (1996), como Areia Litoclástica Média, estéril de componentes biodetríticos e mapeado como banco arenoso (Soares, 2012) (Figuras 4 e 6).

Os recifes representam os arenitos, como os que ocorrem "soldados" à faixa de praia da Praia do Meireles, com áreas submersas e emersas de acordo com o nível da maré. Estas formações rochosas foram identificadas por Morais (1981), como testemunhos dos antigos depósitos sedimentares da Formação Barreiras que foram erodidos pelas ações hidrodinâmicas das ondas e marés ocorridas nos eventos de subida do nível do mar no Pleistoceno.

Alguns destes recifes, quando submersos, são cobertos por sedimentos carbonáticos, e muitas vezes por corais e algas calcárias, como as que ocorrem nas estações \#3, \#11, \#12, \#16 e \#22 (Figura 5).

Os recifes mapeados no extremo NE da área, próximo à estação \#24, conhecido como "Recife do Meireles” (DHN, 2018), estão em cotas batimétricas de -7 metros de profundidade (Figura 6).

Outros recifes mapeados, como os que ocorrem no setor leste do espigão da Praia de Iracema, nas proximidades da Praia do Meireles e das estações \#11, \#12, \#16, \#17, \#21 e \#22, possuem contas variando de -3 a -6 metros, e ocorrem com maior frequência e de forma continua na área.

No setor oeste ao espigão, os recifes ocorrem de maneira isolada, intercalados por substratos arenosos, entre as estações \#1, \#2, \#3 e \#9.

Essa variação de morfologia pode ser visualizada nos quatro perfis batimétricos realizados na área.

O primeiro perfil $(\mathrm{A}-\mathrm{B})$, situado no extremo oeste da área, caracteriza-se por profundidades em torno de -2 metros, próximas à linha de costa, com presença de recifes.

O decaimento do perfil aumenta progressivamente até 650 metros de distância da linha de costa, antes do banco de areia, e possui declividade de $0,26^{\circ}$.

O segundo perfil $(C-D)$, situado a oeste e nas proximidades do espigão da Praia de Iracema, assume comportamento análogo ao anterior, entretanto, sua declividade de $0,16^{\circ}$ se estende até a distância de 1100 metros, e possui morfologia recifal na distância de 700 metros da linha de costa.

O perfil $3(E-F)$, situado no setor leste ao espigão da Praia de Iracema, possui duas estruturas morfológicas recifais, uma na distância de 100 metros, e outra, com altura de cerca de 1 metro, na distância de 400 metros. Sua declividade está em torno de $0,21^{\circ}$. O perfil $4(\mathrm{G}-\mathrm{H})$, localizado no setor extremo leste da área, tem como característica principal o 
destaque aos recifes que estão mais próximos da linha de costa, como o situado na distância de aproximadamente 50 metros, e o situado na distância de 300 metros e que possui 2 metros de altura (Figura 6).
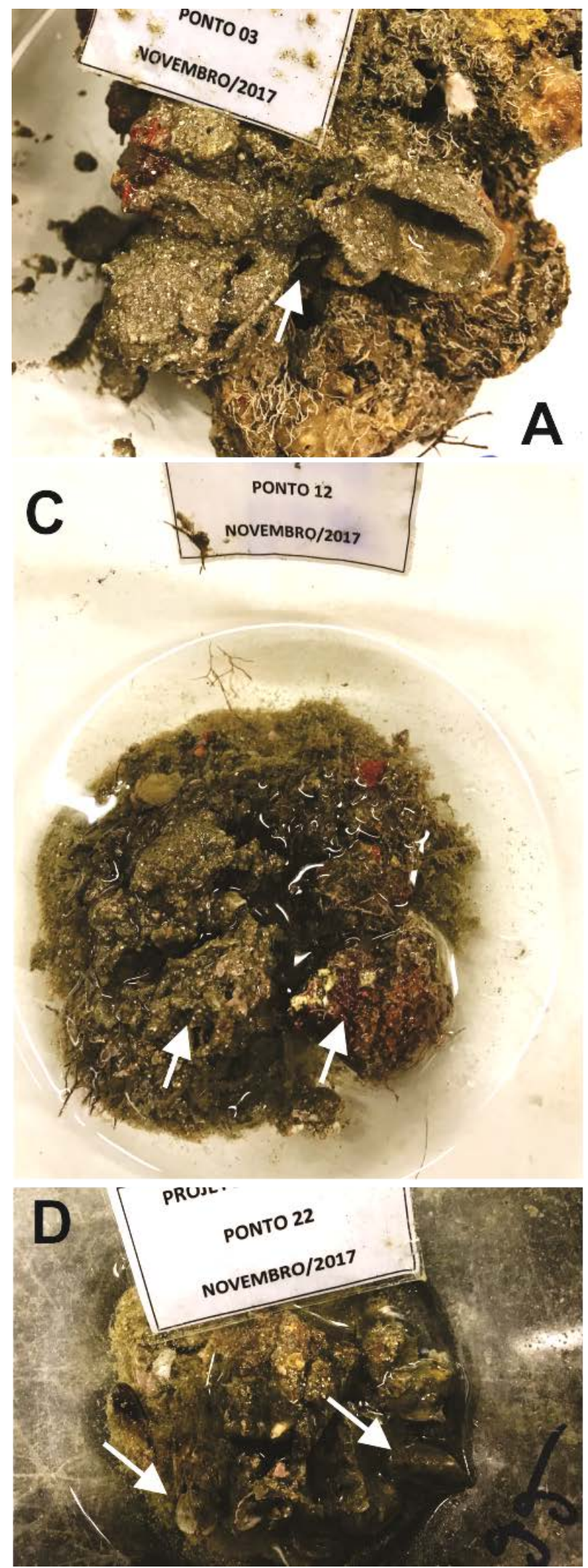

Figura 5. Componentes terrígenos e bióticos nas amostras \#3 (A) - Serpente do Mar (Echinodermata: Ophiuroidea), \#11 (B) - Algas Phaeophyta, \#12; (C) - Algas Phaeophyta e Rodophyta, \#22 (D) - esponjas (Filo Porifera), \#16 (E) Moluscos bivalves (Mollusca: Bivalvia). 

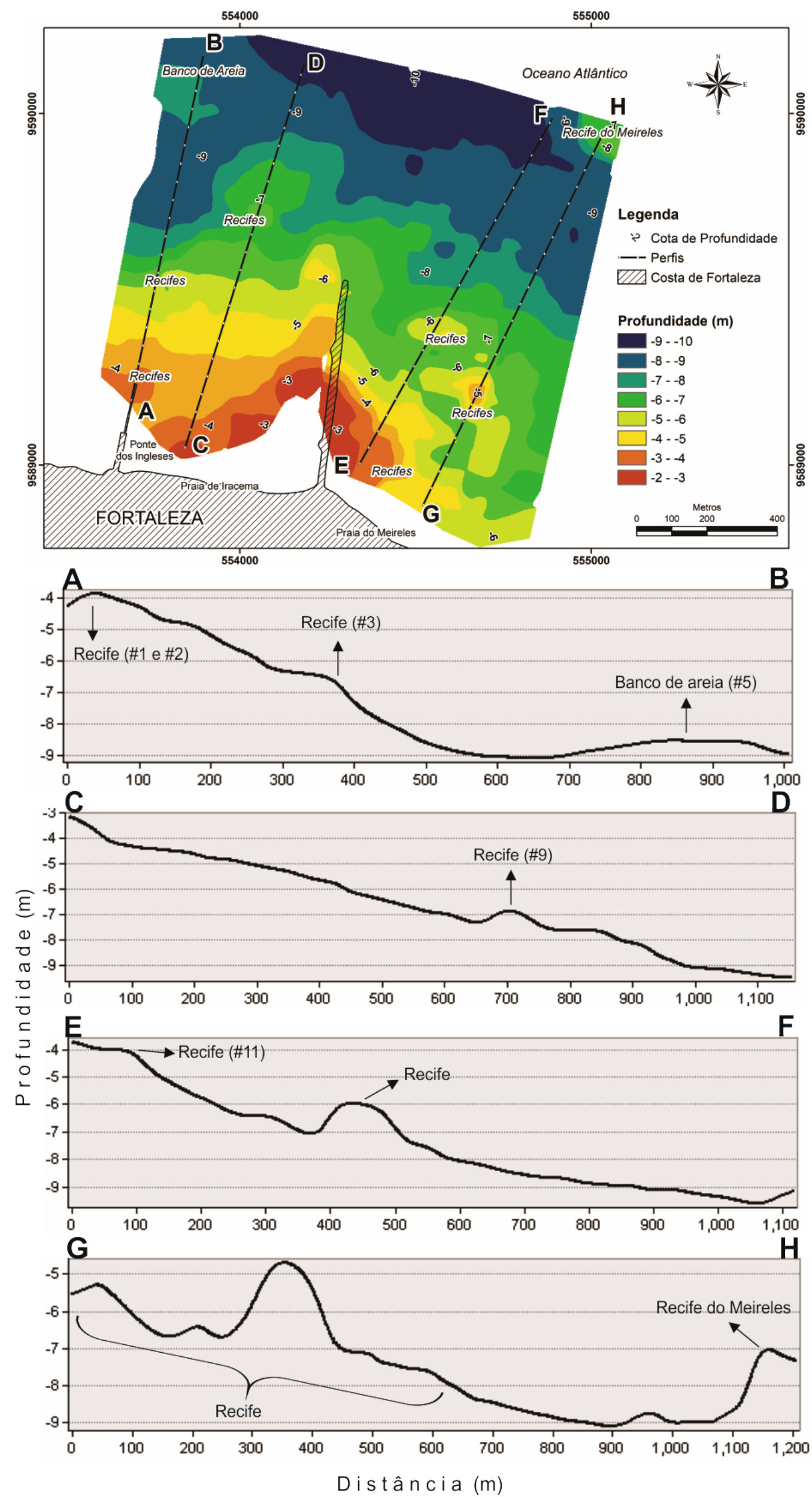

Figura 6- Mapa batimétrico/geomorfológico e perfis com destaques aos recifes submersos. Exagero vertical de 20x. 


\section{Caracterização dos Habitats Marinhos}

A interação entre os dados abióticos (sedimentológicos e batimétricos) dos pontos amostrados no substrato marinho situado na plataforma interna da Praia de Iracema, Fortaleza, com o conjunto de organismos (animais e vegetais) presentes nos sedimentos e/ou fragmentos de carapaças animais permitiu, por intermédio de uma adaptação à classificação proposta pela European Nature Information System (EUNIS, 2012), mapear e classificar quatro habitats marinhos na área de estudo (Tabela 2 e Figura 7).

De acordo com a EUNIS (2012), três correspondem ao Nível 1, Código A, definidos como "Marine Habitats", caracterizados por um substrato majoritariamente inconsolidado. Sendo aqui subdividido em:

Tabela 2. Sistema de classificação das informações pontuais na área de estudo, segundo adaptação da European Nature Information System (EUNIS, 2012).

\begin{tabular}{|c|c|c|c|c|c|}
\hline DOMÍNIOS & 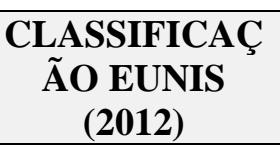 & \multicolumn{2}{|c|}{$\begin{array}{c}\text { COMPONENTES } \\
\text { ABIÓTICOS E BIÓTICOS }\end{array}$} & \multirow{2}{*}{$\begin{array}{c}\text { HABITA } \\
\text { T } \\
\text { A.1 }\end{array}$} & \multirow{2}{*}{$\begin{array}{c}\text { ESTAÇÕES } \\
\text { \#4 e } 5\end{array}$} \\
\hline \multirow{3}{*}{ INCONSOLIDADO } & \multirow{3}{*}{$\begin{array}{l}\text { Marine Habitat } \\
\text { - Nível } 1 \\
\text { - Código A }\end{array}$} & \multirow[t]{2}{*}{ AREIA } & $\begin{array}{c}\text { Média } \\
\text { - Ausência de } \\
\text { biodetritos }\end{array}$ & & \\
\hline & & & $\begin{array}{l}\text { Fina/Muito Fina } \\
\text { - presença de } \\
\text { biodetritos } \\
\text { microscópicos }\end{array}$ & A. 2 & $\begin{array}{c}\# 6,14,15 \\
\# 17,18 \text { e } 24\end{array}$ \\
\hline & & $\begin{array}{l}\text { SILTE/ } \\
\text { ARGILA }\end{array}$ & $\begin{array}{c}\text { Lama } \\
\text { - presença de } \\
\text { biodetritos } \\
\text { microscópicos }\end{array}$ & A. 3 & $\begin{array}{l}\# 7,8,10,13 \\
\#, 19,20 \text { e } 23\end{array}$ \\
\hline CONSOLIDADO & $\begin{array}{l}\text { Littoral Rock } \\
\text { and other hard } \\
\text { substrata } \\
\text { - Nível } 2 \\
\text { - Código A1 } \\
\end{array}$ & $\begin{array}{l}\text { RECIFAL } \\
\text { ROCHOSO }\end{array}$ & $\begin{array}{l}\text { Coral, Rocha dura, } \\
\text { Rocha calcaria, } \\
\text { serpente do mar, } \\
\text { moluscos, esponjas, } \\
\text { algas e poliquetas }\end{array}$ & A1.1 & $\begin{array}{c}\# 3 \\
\# 1,2,9 \text { e } 21 \\
\# 11,12,16 \text { e } \\
22 \\
\end{array}$ \\
\hline
\end{tabular}

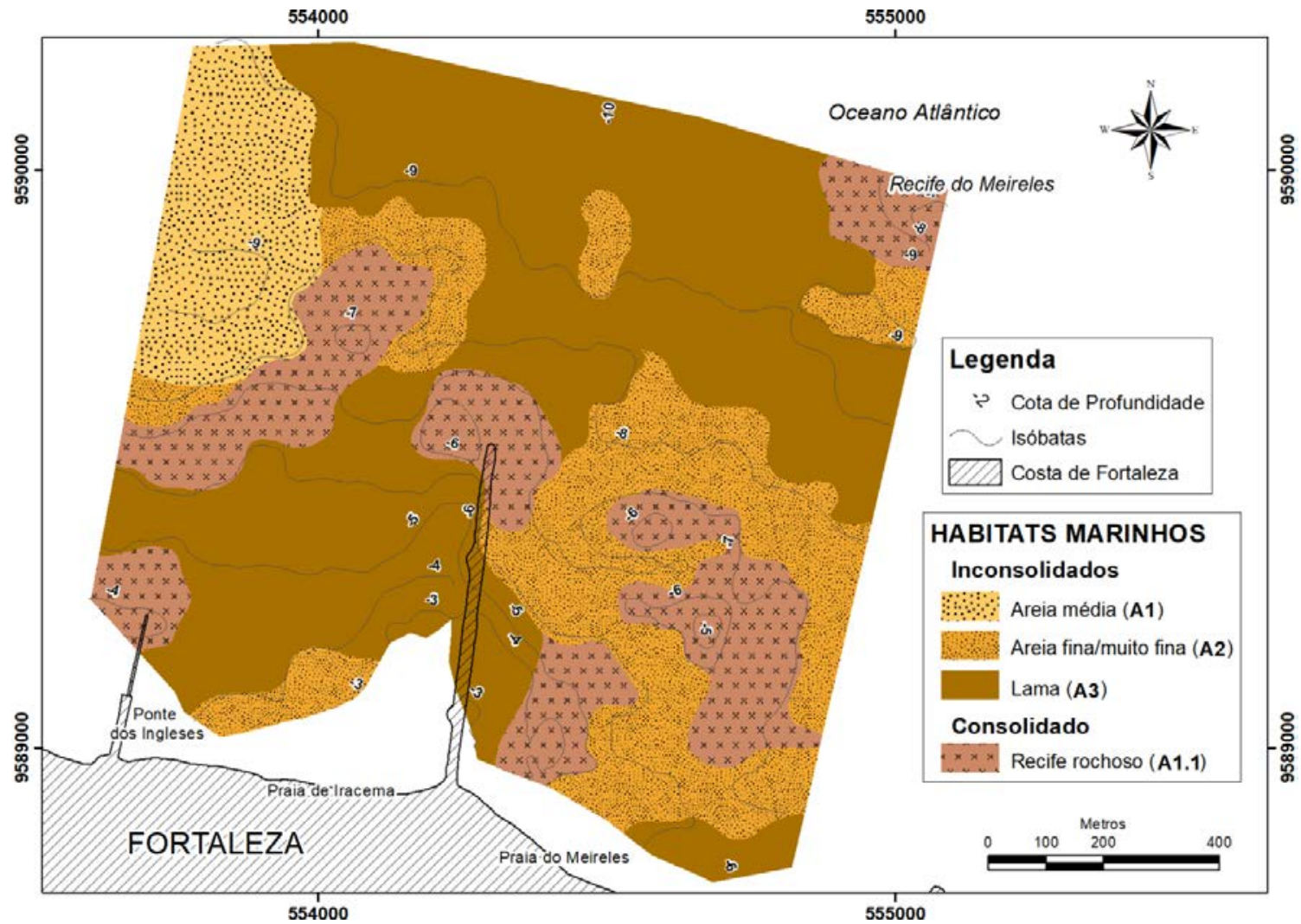

Figura 7. Mapa de distribuição dos habitats marinhos. 
A.1 - Sedimentos do infra litoral, com predominância de areias médias $(>64 \mu \mathrm{m}$ a $<2 \mathrm{~mm}$ ), sem a presença notória de material biodetrítico, sendo, associados às feições morfológicas mapeadas por Soares (2012) como “Bancos Arenosos”. Estações: \#4 e 5.

A.2 - Sedimentos do infra litoral, constituídos por areias predominantemente siliciclásticas, com granulometria variando de fina a muito fina. Notando ocorrências esparsas de fragmentos biodetríticos (fragmentos de algas (Filos Rhodophyta e Phaeophyta)). Estações: \#6, 14, 15, 17, 18 e 24.

A.3 - Sedimentos do infra litoral, com predomínio da lama arenosa, com a ocorrência de elevado número de algas macroscópicas das divisões Rodophyta e Phaeophyta, assim como esponjas vermelhas do Filo Porifera e tubos membranosos pertencentes ao Filo Chordata da classe Ascidiacea. Sendo, verificada a presença de fragmentos biodetríticos microscópicos, caracterizados por carapaças de foraminíferos, espículas de esponjas, fragmentos de briozoários, ouriços e crustáceos. Estações: \#7, 8, 10, 13, 19, 20 e 23.

As demais amostras, quando possível de serem coletadas, foram agrupadas no Nível 2 da EUNIS (2012), Código A1, denominada de "Littoral Rock andother hard substrata", caracterizadas por um substrato duro/rochoso:

A1.1 - Afloramento pontual de rocha do infra litoral, bordejado por sedimentos inconsolidados, com rica abundancia biológica. Com a presença de "Serpentes do Mar" do Filo Echinodermata - Classe Ophiuroidea. Também foram notados exemplares macroscópicos do Filo Mollusca da classe Bivalvia, esponjas do Filo Porifera, algas calcárias (rodolitos) e Filo Annelida da classe Polychaeta. Estação \#3.
Rocha do Infra litoral, presença de substrato formado por rochas consolidadas, na área de arrebentação. Pontos não amostrados em função da rigidez do substrato. Provavelmente constituído por uma associação de algas vermelhas (Rodofíceas - Rodophyta) e pardas (Feofíceas - Phaeophyta), esponjas (Filo Porífera). Estações: \#1, 2, 9 e 21. E sedimentos do infra litoral, caracterizado por granulometria de diâmetro superior a $2 \mathrm{~mm}$ (cascalhos), marcados por fragmentos de carapaças de organismos marinhos. Presença de rodolitos (algas vermelhas - calcárias), esponjas (Filo Porifera) e macro algas como ramos de algas vermelhas (Rodofíceas - Rodophyta) e pardas (Feofíceas - Phaeophyta). Estações: \#11, 12, 16 e 22.

A distribuição espacial dos habitats mapeados pode ser visualizada na Figura 7, sendo destacados os dois grandes domínios: Inconsolidados e Consolidados.

As áreas caracterizadas como substrato Inconsolidado foram subdivididas em: Areias, cujos grãos foram classificados como médios, finos e muito finos, com presença e/ou ausência de fragmentos biodetríticos, e Lamas, onde a percentagem de silte + argila variaram de 25 a $75 \%$, com teores de carbonato de cálcio (CaCO3) < 30\%.

As áreas mapeadas como Consolidados correspondem aos substratos amostrados caracterizados por uma natureza rochosa (\#3), pelos pontos onde a draga não conseguiu realizar a coleta em função de um substrato consolidado (rochoso) (\#1, 2, 9 e 21), assim como nas estações \#11, 12, 16 e 22, onde as amostras foram caracterizadas por pequena quantidade de areias finas e representativa percentagem de fragmentos cascalhosos de algas.

\section{CONCLUSÕES}

O mapeamento dos habitats marinhos corresponde a uma pesquisa ainda muito complexa, principalmente quando associado à vasta diversificação nos componentes biológicos que habitam as águas e substratos marinhos.

A intenção inicial da presente pesquisa foi alcançada quando se realizou uma interação entre os componentes abióticos (sedimentologia e batimetria) e os componentes bióticos (animais e vegetais) identificados no fundo marinho.
De maneira geral, a área caracterizou-se por trechos constituídos por um substrato inconsolidado e por substrato consolidado. Dentro desse contexto foram mapeados cinco habitats marinhos.

O substrato inconsolidado, dependendo das atuantes oceanográficas, foi caracterizado por depósitos de grãos minerais inorgânicos, principalmente por grãos de quartzo (SiO2), de diâmetros variando de areia média $(+2,0<\varnothing>$ $+1,0)$, areia fina $(+3,0<\varnothing>+2,0)$, areia muito fina $(+4,0<\varnothing>+3,0)$ e de frações constituídas 
por silte + argila $(\varnothing>4,0)$ (Wentworth1922, in: Suguio, 1973).

Nesse contexto foi possível isolar áreas classificadas por Larsounner (1977) e modificadas por Dias (1996) como Areias Litoclásticas Médias, totalmente estéreis de constituintes orgânicos/biodetritos, com teores de carbonato de cálcio < 10\%, estações \#4 e \#5. Assim como áreas com substrato classificados por Areias Litoclásticas Finas e muito Finas (\#6, 14, 15, 17, 18 e 24), e Lama Terrígena Arenosa $(\# 7,8,10,13,19,20$ e 23), com teores de carbonato de cálcio variando de 10 a 30\%, e quantidade representativa de biodetritos (e.g. Fragmento de concha de bivalve e espículas de esponjas da estação \#7; Foraminíferos, fragmentos de bivalves e crustáceos da estação \#8; e Fragmento de espinho de ouriço da estação \#10). Sendo, portanto, mapeados três habitats marinhos nesse domínio de substrato inconsolidado (A.1, A.2 e A.3).

Em núcleos isolados distribuídos no domínio do substrato inconsolidado foram mapeados trechos onde o substrato marinho caracteriza-se por ser consolidado, podendo representar antigos depósitos sedimentares da Formação Barreiras que foram erodidos pelas ações hidrodinâmicas (ondas e marés) ocorridas nos eventos de subida do nível do mar durante o Pleistoceno (Morais, 1981), ou pelo desenvolvimento de corais e de algas calcarias.

Esses depósitos funcionam como meio de fixação para organismos marinhos, ocasionando, portanto, um enriquecimento da biota marinha, que se adaptam e sobrevivem. Alguns destes recifes, quando submersos, são cobertos por sedimentos carbonáticos, e muitas vezes por corais e algas calcárias, com teores de carbonato de cálcio de $>20 \%$. A estação \#3 caracteriza-se por uma abundância de organismos (e.g. "Serpentes do Mar" (Filo Echinodermata - Classe Ophiuroidea) e exemplares macroscópicos do Filo Mollusca (Classe Bivalvia), Esponjas (Filo Porifera), Algas Calcárias (rodolitos) e Filo Annelida (Classe Polychaeta)). As estações \#11 e \#12 foram marcadas pela presença de fragmentos de algas (Divisão Rodophyta e Phaeophyta). A estação \#16, pela presença de rodolitos (algas vermelhas - calcárias), esponjas (Filo Porifera) e macro algas vermelhas (Rodophyta) e pardas (Phaeophyta). Na estação \#22 foi possível identificar rodolitos (algas vermelhas calcárias), esponjas (Filo Porifera) e macro algas vermelhas (Rodophyta) e pardas (Phaeophyta). Nesse domínio de substrato consolidado foi possível individualizar os habitats marinhos - A1.1.

Desta forma o mapeamento dos habitats marinhos, apresentado pela primeira vez em escala de detalhe na área estudada, propõe melhor compreensão da importância da preservação destas áreas para a manutenção da vida marinha costeira e de plataforma rasa.

\section{AGRADECIMENTOS}

Agradecemos aos Laboratórios de Oceanografia Geológica (LOG) e Dinâmica Costeira (Labdic) do Instituto de Ciências do Mar - Labomar, da Universidade Federal do Ceará (UFC), pelo apoio na infraestrutura laboratorial. Ao Conselho Nacional de Desenvolvimento Científico e Tecnológico CNPq pela Bolsa de Pesquisa à primeira autora, Proc. $n^{0}$ : 309140/2018-8. Ao Projeto de Geodiversidade Impactos Sócio ambientalistas no Sistema Praia-Plataforma da Costa Oeste do Ceará - PRONEX - FUNCAP - CNPq.

\section{REFERÊNCIAS}

BEGON, M.; HARPER, J.L.; TOWNSEND, C.R. Ecology: individuals, population sand communities. Blackwell Scientific Publications; 1990.

BRASIL. Ministério do Meio Ambiente. Projeto Orla: fundamentos para a gestão integrada. Brasília, DF: Ministério do Meio Ambiente, Ministério do Planejamento, Orçamento e Gestão, 2006.

CATANZARO, L.F. Distribuição de sedimentos e características de ecocaráter $(7,0 \mathrm{kHz})$ na Baía de Guanabara - RJ, Rio de Janeiro, 2002. Dissertação (Mestrado) - Departamento de Geologia, Universidade Federal Fluminense, Niterói.

COLEMAN, K.D.S.; JENKINSON, G.J.; CROCKER, P.R.; GRACE, J.; KLÍR, M.; KÖRSCHENS, P.R.; POULTON, D.D.; RICHTER Simulate ting trends in soil organic carbon in long-term experiment tussling Roth C-26.3. P. Smith, D.S. Powlson, J.U. Smith, E.T. Elliott (Eds.), Geoderma, v. 81, p. 29-44, 1997.

CONNOR, D.W.; ALLEN, J.H.; GOLDING, N.; HOWELL, K.L.; LIEBERKNECHT, L.M.; NORTHEN, K.O.; REKER, J.B. The marine habitat classification for Britain and Ireland version 04.05 introduction. Joint Nature Conservation Committee. Institute of Coastaland Estuarin Estudies, Universityof Hull, 2004.

COUTINHO, P.N. Levantamento do Estado de Arte da Pesquisa dos Recursos Vivos Marinhos do Brasil. Projeto REVIZEE - Oceanografia Geológica (Costa Nordeste): FEMAR/SECIRM: 78p, 1996.

DAVIES, B.E. Loss-on-ignition as an estimate of soil organic matter. Aoil. Sci. Soc. Amer. Proc., v. 38, 1974. 
DHN. Diretoria de Hidrografia e Navegação - disp. em: https:/www.marinha.mil.br/chm/dados-do-segnav-cartasnauticas/cartas-nauticas. CARTA Náutica $\mathrm{N}^{\circ}$ 701. Acesso em:14/08/2018.

DIAS, G.T.M. Classificação de sedimentos marinhos inconsolidados - proposta de representação em cartas sedimentológicas. In: Congresso Brasileiro de Geologia, 39, Salvador, 1996. Anais...Salvador: Sociedade Brasileira de Geologia, 1996.

DIAS, G.T.M. \& MEDEIROS, M. F. Proposta de metodologia para elaboração de cartas sedimentológicas. In: ABEQUA, X, Guarapari, 2005. Anais... Guarapari: Associação Brasileira de Estudos do Quaternário.

DIAS G.T.M.; ROBRINI M.; FREIRE Anais G.S.F.; FIGUEIREDO JR A.G. Cartas de Sedimento de Fundo. Áreas da Oceanografia Geológica (Sul, Central, Nordeste e Norte) - Programa de Avaliação ao do Potencial Sustentável dos recursos vivos na Zona Econômica Exclusiva. REVIZEE, 2004a. CD-ROM de Cartas Sedimentológicas.

DIAZ, R.J.; SOLAN, M.; VALENTE, R.M. A reviewof approaches for classifyingbenthic habitats andevaluating habitat quality. Journal of Environmental Management, v. 73, p. 165-181, 2004b.

EUNIS. EUROPEAN NATURE INFORMATION SYSTEM (EUNIS). In: https://eunis.eea.europa.eu/habitats.jsp. 2007, revisada em 2012.

FREIRE, G.S.S. Geologia marinha da plataforma continental do Ceará. Fortaleza, 1985. 132p. Dissertação (Mestrado) Universidade Federal de Pernambuco, Centro de Tecnologia, Departamento de Geologia,

FREIRE, G.S.S. \& CALVALCANTI, V.M.M. A Cobertura Sedimentar Quaternária da Plataforma Continental do Estado do Ceará. Fortaleza, 42p, 1998.

FREIRE, G.S.S.; COUTINHO, P.N.; MORAIS, J.O Sedimentos superficiais da plataforma continental do Ceará. In: SIMPÓSIO DE GEOLOGIA DO NORDESTE, 11, Natal, 1984. Atas....Natal: Sociedade Brasileira de Geologia.

FREIRE, G.S.S.; MAIA, L.P.; CAVALCANTI, V.M.M.; FONTELES, M.L. Distribuição dos minerais pesados na plataforma continental do Estado do Ceará (costa leste). In: SIMPÓSIO DE GEOLOGIA DO NORDESTE, Natal, 1993. Atas....Natal: Sociedade Brasileira de Geologia, 1993.

GREENE, H.G.; BIZZARRO, J.J.; O’CONNELL, V.M.; ANDBRYLINSKY, C.K. Construction of digital potential marine benthic habitat maps using a coded classifications scheme and its application. In: Mapping the Seafloor for Habitat Characterization, Ed. B.J. Todd; H.G. Greene. Geological Association of Canada, St John's, Newfoundland, Canada, p. 141-155, 2007.

IPECE. Instituto de Pesquisa e Estratégia Econômica do Ceará. In: Disp. na Internet: https://www.ipece.ce.gov.br. Acesso em 16abr2018.

KEMPF, M.; COUTINHO, P.N.; MORAIS, J.O. Plataforma Continental do Norte e Nordeste do Brasil, nota preliminar sobre a Natureza do Fundo. Trabalho Oceanográfico. Universidade federal de Pernambuco, v. 9/11, p. 9-26, 1970.

LARSONNEUR, C. La caryographie de's dépots meubles sur le plateau continental français: méthode mise du points et utilisée en Manche. 1977. Journal Redi oceanog, vol. 2, pag. 34-39

LIMA FLHO, R.P.; GASTÃO, F.G.C.; CASTELO BRANCO, M.P.N. PINHEIRO, L.S. Caracterização Geomorfológica e Sedimentológica da Plataforma Continental Interna de Fortaleza. In: SINAGEO, PAISAGEM E GEODIVERSIDADE, XII Crato, 2018 Anais...Crato: Simpósio Nacional de Geomorfologia. 2018.
MAIA, L.P. FREIRE, G.S.S.; PESSOA, P.R.S.; RODRIGUES, A.C.B.; MAGALHÃES, S.H.O.; ARAÚJO, P.S.A. Transporte de sedimentos na região costeira do Ceará. Fluxo de sedimentos e caracterização granulométrica. In: SIMPÓSIO DE GEOLOGIA DO NORDESTE, XVII, Fortaleza, 1997. Anais...Fortaleza: Sociedade Brasileira de Geologia, v. 15, p. 182-192, 1997.

MAIA, L.P.; VASCONCELOS, F.P.; MONTEIRO, L.H.V.; REBOUÇAS, R.B.M.; MARTINS, M.B. Projeto executivo emergencial de proteção/recuperação da praia de Iracema. Prefeitura Municipal de Fortaleza. Programa de Recuperação do Litoral da Cidade. Laboratório de Ciências do Mar, Universidade Federal do Ceará, 2008 (Documento restrito).

MÖLLER, P.; PIHL, L.; ROSENBERG, R. Benthic faunal energy flow and biological interaction in some shallow marine soft bottom habitats. Mar. Ecol. Prog. Ser., v. 27, p. 109-121, 1985.

MONTEIRO, L.H.U. Feições Superficiais da Plataforma Continental Cearense entre o Litoral de Fortaleza e Icapuí. Rio Claro, 2011. Tese (Doutoramento em Geociências) Universidade Estadual Paulista.

MORAIS, J. O. Processo de assoreamento do Porto de Mucuripe. Arquivo Ciências do Mar, v. 12, p. 139-149, 1972.

MORAIS, J.O. Evolução sedimentológica da Enseada do Mucuripe. Arquivo de Ciências do Mar, Fortaleza, v. 21, n. (1/2), p. 19 - 46, 1981.

REBOUÇAS, R.B.M. A Influência portuária no modelado e reconfiguração da orla. O caso do Porto do Mucuripe e Praia de Serviluz, Fortaleza, Ceará. Fortaleza, 2010. Tese (Mestrado) - Universidade Estadual do Ceará.

SECCHIN, N.A. Mapeamento de Habitats Marinhos na Plataforma de Abrolhos. Vitória, 2011. 71 p. Dissertação (Mestrado) - Universidade Federal do Espirito Santo.

SOARES, R.C. Evolução e caracterização do banco sedimentar de Fortaleza, Ceará, Brasil. 2012. Fortaleza, 115 p. Dissertação (Mestrado em Ciências Marinhas Tropicais) - Instituto de Ciências do Mar, Universidade Federal do Ceará.

SOARES, R.C.; MORAIS, J.O. Balanço sedimentar do aterro da Praia de Iracema. Fortaleza- Ceará. In: SIMPÓSIO DE GESTÃO INTEGRADA DA ZONA COSTEIRA, III, Fortaleza, 2009

SOARES, R.S. Novas proposições metodológicas para o calcímetro de Bernard e caracterização dos sedimentos marinhos do Espírito Santo. 2017. Fortaleza. 89 p. Dissertação (Mestrado em Ciências Marinhas Tropicais) Universidade Federal do Ceará.

SUGUIO, K. Introdução a sedimentologia. São Paulo. Ed. Edgard Blucher. EDUSP, 1973, 317 p. 